\title{
Neutrino electron scattering and left-right symmetry: future tests
}

\author{
O. G. Miranda* V. Semikoz ${ }^{\dagger}$ and José W. F. Valle ${ }^{\ddagger}$ a \\ ${ }^{a}$ Instituto de Física Corpuscular - C.S.I.C. \\ Departament de Física Teòrica, Universitat de València \\ 46100 Burjassot, València, Spain
}

\begin{abstract}
Low-energy high-resolution neutrino-electron scattering experiments may play an important role in testing the gauge structure of the electroweak interaction. We propose the use of radioactive neutrino sources (e.g. $\left.{ }^{51} \mathrm{Cr}\right)$ in underground experiments such as BOREXINO and HELLAZ as a probe of the weak neutral current structure. As an illustration, we display the sensitivity of these detectors in testing the possible existence of right-handed weak neutral currents.
\end{abstract}

Probing the gauge structure of the electroweak interaction using accelerators or reactor neutrinos has already been common practice for a number of years. Accelerators provide mostly muon neutrino beams and their precision in determining the neutral current parameters is well below present LEP accuracy. The first observation of $\nu_{e} e \rightarrow \nu_{e} e$ scattering at LAMPF [1] resulted in a total of $236 \pm 35$ events [2] leading to a total cross section of $(10.0 \pm 1.5 \pm 0.9) \times 10^{-45} \mathrm{~cm}^{2} \times E_{\nu}(\mathrm{MeV})$ for a neutrino mean energy of $30 \mathrm{MeV}$. This measurement showed that the interference term between neutral and charged currents is negative, as predicted by the standard model. However, it is very hard to achieve a precision measurement that is able to probe very accurately the parameters of the weak neutral current. As for reactor experiments, one is limited by the accuracy of electron anti-neutrino fluxes as well as by geometry.

Here we suggest the possibility of studying $\nu_{e} e \rightarrow \nu_{e} e$ scattering from terrestrial neutrino sources with improved statistics. A similar idea has been proposed as a test of non-standard neutrino electro-magnetic properties, such as magnetic moments [3]. In contrast to reactor experiments, a small radioactive isotope source can be surrounded by gas or liquid scintillator detectors with full geometrical coverage. Here we

\footnotetext{
* On leave from Departamento de Física CINVESTAV-IPN

${ }^{\dagger}$ On sabbatical leave from Izmiran, Moscow

${ }^{\ddagger}$ http://neutrinos.uv.es
}

show how a low-energy high-resolution experiment may play a role in testing the structure of the neutral current weak interaction. The ingredients for doing such experiments are either already available (e. g. the chromium source has already been used for calibrating the GALLEX experiment [4]) or under investigation (NaI detectors have already been used in dark matter searches [5] and the BOREXINO and HELLAZ [6.7] detectors have been extensively discussed). These detectors should reach good energy resolution and relatively low threshold. Some cryogenic detectors have also been mentioned in this context [8].

Here we explicitly determine the sensitivity of these radioactive neutrino source experiments as probes of the gauge structure of the electroweak interaction, with special emphasis on left-right symmetric extensions of the standard electroweak theory.

We assume a generic electroweak gauge model in which the main contributions to the neutrino electron scattering cross section arise from the exchange of charged and neutral intermediate vector bosons, i.e. from charged (CC) and neutral currents (NC).

The charged current amplitude for the $\nu_{e} e \rightarrow$ $\nu_{e} e$ process can be written, after a Fierz transformation as

$\mathcal{M}_{\mathcal{C C}}=\sqrt{2} G_{F} \bar{\nu} \gamma^{\nu}\left(1-\gamma^{5}\right) \nu \bar{e} \gamma_{\mu} c_{L} \frac{1-\gamma^{5}}{2} e$. 
For the specific case of the Standard Model (SM) we have $c_{L}=1$.

On the other hand, the neutral current contribution to the amplitude for the $\nu_{e} e \rightarrow \nu_{e} e$ process can be given as

$$
\begin{aligned}
\mathcal{M}_{\mathcal{N C}} & =\sqrt{2} G_{F} \bar{\nu} \gamma^{\mu}\left(1-\gamma^{5}\right) \nu\left\{\overline { e } \gamma _ { \mu } \left[g_{L} \frac{1-\gamma^{5}}{2}+\right.\right. \\
& \left.\left.+g_{R} \frac{1+\gamma^{5}}{2}\right]\right\} e .
\end{aligned}
$$

In this case the tree level (SM) prediction is $g_{L, R}=\frac{1}{2}\left(g_{V} \pm g_{A}\right), g_{V}=-1 / 2+2 \operatorname{Sin}^{2} \theta_{W}$ and $g_{A}=-1 / 2$. The values of these coupling constants have been well measured from $e^{+} e^{-} \rightarrow$ $l^{+} l^{-}$at high energies by the LEP Collaborations. A combined fit from LEP results at the $\mathrm{Z}$ peak gives [9] $g_{V}=-0.03805 \pm 0.00059$ and $g_{A}=-0.50098 \pm 0.00033$. These results have given strong constraints on right-handed neutral currents. Radiative electroweak corrections for the SM at low energies were recently computed [10] and are included in computations.

The $\nu_{e} e \rightarrow \nu_{e} e$ differential cross section following from (11) and (2) in terms of $T / \omega_{1}$ is given by

$$
\begin{aligned}
\frac{d \sigma}{d T} & =\frac{2 m_{e} G_{F}^{2}}{\pi}\left\{\left(g_{L}+c_{L}\right)^{2}+g_{R}^{2}\left(1-\frac{T}{\omega_{1}}\right)^{2}-\right. \\
& \left.-\left(g_{L}+c_{L}\right) g_{R} \frac{m_{e}}{\omega_{1}} \frac{T}{\omega_{1}}\right\}
\end{aligned}
$$

Here, $T$ is the recoil electron energy, and $\omega_{1}$ is the neutrino energy; therefore $T / \omega_{1}<1$ for any value of $\omega_{1}$. It is also important to note that we have in this expression the ratio $m_{e} / \omega_{1}$, which is important for low energies and negligible for accelerator neutrino energies. All terms in this cross section are potentially sensitive to the right-handed current admixtures. As we will see neutrino-electron scattering experiments with sufficiently low energies and high resolution may provide a novel way to test for the presence of right-handed neutral currents.

We now turn to a brief discussion on the models based on the Left-Right Symmetric gauge group $S U(2)_{L} \otimes S U(2)_{R} \otimes U(1)_{B-L}[11,12$. These are attractive since they offer the possibility of incorporating parity violation on the same footing as gauge symmetry breaking, instead of by hand as in the SM. The Lagrangian for neutral currents in the left-right symmetric model (LRSM) is given by

$$
-\mathcal{L}=g J_{T_{L}}^{3^{\mu}} W_{L}^{3^{\mu}}+g J_{T_{R}}^{3^{\mu}} W_{R}^{3^{\mu}}+g^{\prime} J_{Y}^{\mu} B^{3^{\mu}}
$$

where $J_{T_{L, R}}^{3^{\mu}}=\bar{\psi}_{L, R} \gamma^{\mu} T_{L, R}^{3} \psi_{L, R}$ and $T_{L, R}^{3}$ is the third isospin component.

After applying the corresponding neutral gauge boson diagonalization matrix to get the mass eigenstates we have

$$
\begin{aligned}
\mathcal{L} & =g s_{W}\left[J_{\mathrm{em}} A-\frac{1}{c_{W}}\left(a_{1} J_{L}^{Z}+b_{1} J_{R}^{Z}\right) Z_{1}+\right. \\
& \left.+\frac{1}{c_{W}}\left(a_{2} J_{L}^{Z}+b_{2} J_{R}^{Z}\right) Z_{2}\right]
\end{aligned}
$$

where $J_{L, R}=J_{L, R}^{3}-\sin ^{2} \theta_{W} J_{e m}$ and

$$
\begin{aligned}
a_{1} & =s_{W} \frac{s_{\phi}}{r_{W}}-\frac{c_{\phi}}{s_{W}}, & b_{1} & =s_{\phi} \frac{c_{W}^{2}}{s_{W} r_{W}}, \\
a_{2}=\frac{s_{W} c_{\phi}}{r_{W}}+\frac{s_{\phi}}{s_{W}} & , & b_{2} & =\frac{c_{\phi} c_{W}^{2}}{s_{W} r_{W}} .
\end{aligned}
$$

Here $s_{W}, c_{W}$ and $r_{W}$ is a shorthand notation for $\sin \theta_{W}, \cos \theta_{W}$ and $\sqrt{\cos 2 \theta_{W}}$; and $s_{\phi}, c_{\phi}$ for $\sin \phi$, $\cos \phi$ with $\phi$ the mixing angle between $Z_{L}$ and $Z_{R}$.

In the low energy limit, the $\nu_{e} e \rightarrow \nu_{e} e$ amplitude is given, for left handed $\nu_{e}$ by

$$
\mathcal{M}_{N C}^{L R}=\frac{8 G_{F}}{\sqrt{2}}\left[A J_{L}^{\nu} J_{L}^{e}+B J_{L}^{\nu} J_{R}^{e}\right]
$$

with

$$
\begin{aligned}
& A=s_{W}^{2}\left(a_{1}^{2}+\gamma a_{2}^{2}\right) \\
& B=s_{W}^{2}\left(a_{1} b_{1}+\gamma a_{2} b_{2}\right)
\end{aligned}
$$

and $\gamma=\left(\frac{M_{Z_{1}}}{M_{Z_{2}}}\right)^{2}$.

Comparing the amplitude in Eq. (7) with the one shown in Eq. (2) we can identify the expressions for the neutral current constants in this model as

$$
\begin{aligned}
& g_{L}=A \frac{1}{2}\left(g_{V}+g_{A}\right)+B \frac{1}{2}\left(g_{V}-g_{A}\right) \\
& g_{R}=A \frac{1}{2}\left(g_{V}-g_{A}\right)+B \frac{1}{2}\left(g_{V}+g_{A}\right) .
\end{aligned}
$$

The dependence on the LRSM parameters is contained in $A$ and $B$. Therefore, the differential 
cross section in the LRSM will be that of Eq (3) with the values for $g_{L}$ and $g_{R}$ given by Eq. (8).

Note that $\nu_{e} e \rightarrow \nu_{e} e$ scattering is not sensitive to right-handed charged currents because the interference term between the corresponding amplitude and the SM one is suppressed either by the neutrino mass (Dirac case) or by the mixing with the heavy neutrinos (Majorana case, seesaw model). In fact, this this just an example of the general situation that one finds when trying to constrain charged-current parameters via purely leptonic processes (see [13] and 14]).

Neutral current couplings have been measured with a great accuracy at the $\mathrm{Z}$ peak in LEP and they give strong bounds on the mixing angle $\phi$ between massive neutral gauge bosons (the allowed range being $-0.015<\phi<0.002$ ) [15]. On the other hand the mass of the additional gauge boson is constrained by direct searches or by electroweak fits [16]. In the latter case the constraint on the mass of the extra neutral gauge boson translates into $\gamma \leq 0.055$ at $95 \%$ C. L. [16]. Equivalent constraints coming from neutrino experiments were derived by the CHARM II Collaboration [17] for $\nu_{\mu} e \rightarrow \nu_{\mu} e$ scattering, leading to $\gamma \leq 0.130$ at $95 \%$ C. L., far worse than the one obtained in a global electroweak fit.

Here we want to show that new low-energy high-precision $\nu_{e} e \rightarrow \nu_{e} e$ scattering experiments could improve this constraint. Two ingredients are required for this kind of experiment: a strong low-energy electron-neutrino source and a highprecision detector. Here we focus on the potential of radioactive neutrino sources (e.g. ${ }^{51} \mathrm{Cr}$ ) in underground detectors as a probe of the weak neutral current structure. The first strong low-energy neutrino source has been recently prepared for the calibration of the GALLEX neutrino experiment [4]. This was a ${ }^{51} \mathrm{Cr}$ source with an activity of $1.67 \pm 0.03 \mathrm{MCi}$. The main neutrino lines in this source correspond to $0.746 \mathrm{MeV}(81 \%)$ and 0.751 $\mathrm{MeV}(9 \%)$.

At present $\nu_{e} e \rightarrow \nu_{e} e$ scattering has not been measured at energies below $1 \mathrm{MeV}$. However, there are several proposals in this direction. Here we concentrate in BOREXINO and HELLAZ. These will be sensitive to the desired range of elec- tron recoil energy. The expected energy thresholds are $250 \mathrm{KeV}$ and $100 \mathrm{KeV}$ respectively. As for the energy resolution, BOREXINO should reach $12 \%$ at $250 \mathrm{KeV}$ and $8 \%$ at $500 \mathrm{KeV}$, so we take bins of $50 \mathrm{KeV}$ width. In the case of HELLAZ a mean energy resolution of few percent is envisaged; therefore, we consider $10 \mathrm{KeV}$ bins. Thus BOREXINO and HELLAZ can detect the two main lines of the ${ }^{51} \mathrm{Cr}$. Here we estimate the expected differential number of events both for the SM and the left-right symmetric model, using the expression

$$
\begin{aligned}
\frac{d N}{d T} & =\Phi N_{e} \Delta t\left\{0.81\left(\frac{d \sigma}{d T}\right)_{E_{\nu}=746 \mathrm{KeV}}+\right. \\
& \left.+0.09\left(\frac{d \sigma}{d T}\right)_{E_{\nu}=751 \mathrm{KeV}}\right\} .
\end{aligned}
$$

where we have assumed that these detectors surround the neutrino source, as in the case of GALLEX [- [ith with an exposure time $\Delta t$ of twenty days.

In order to have an idea of the expected sensitivity on $\gamma$ as a function of the hypothetical measurement, we must guess the measurement numbers including their errors, as well as the energy resolution attained in the detectors.

Let us assume that the detectors will measure the Standard Model prediction with a hypothetical error $\sigma_{N_{i}}$ for bin $i$. In this case we can determine the corresponding constraint on $\gamma$ as a function of $\sigma_{N_{i}}$. In Fig 1 we display the constraint at $95 \% \mathrm{C}$. L. which can be attained on the ratio $\gamma$ as a function of $\sigma_{N_{i}}$ (in percent).

Due to the high expected number of events in BOREXINO, the expected statistical error for $N_{i}$ is of the order of $0.5 \%$. Therefore, the constraining power in this case is mostly dependent on the systematic error. If the systematic error can be kept at the level of $3 \%$, then it will be possible to get a bound on $\gamma$ of the same order as obtained by CHARM II for the case of $\nu_{\mu}$ scattering.

In contrast for the case of HELLAZ we expect to achieve a better energy resolution, leading to a lower slope than the previous case, as displayed in the figure. Thus in this case one correspondingly obtains a better sensitivity to the parameter $\gamma$.

Another possibility is the LAMA proposal [5]. 


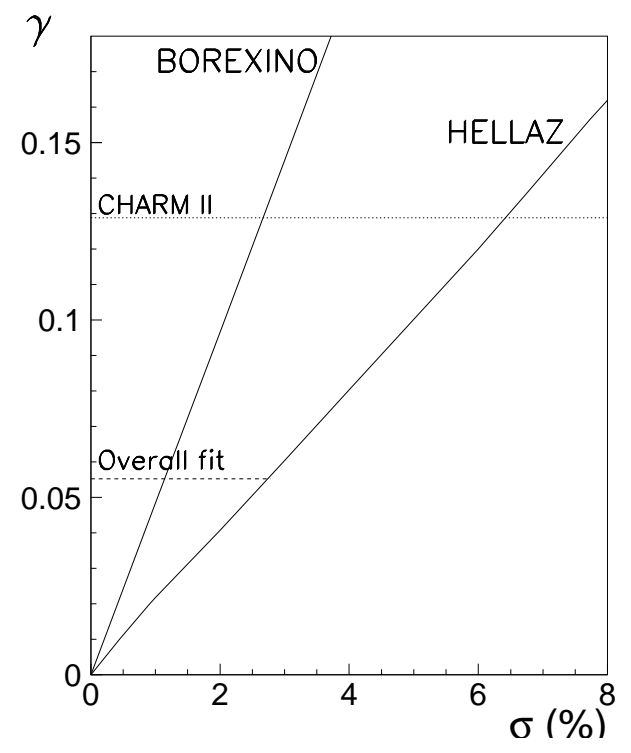

Figure 1. Constraining power on $\gamma$ for different experiments depending on the experimental error

This experiment proposes to use a one ton $\mathrm{NaI}$ detector with similar characteristics to the 100 $\mathrm{Kg}$ version already used for dark matter searches. The proposed source is an anti-neutrino ${ }^{147} \mathrm{Pm}$ source that could reach an activity of $5 \mathrm{MCi}$ and a half-life of 2.6 years. In contrast to the ${ }^{51} \mathrm{Cr}$ this source has a continuous spectrum instead of discrete lines. The maximum neutrino energy will be $234.7 \mathrm{KeV}$. In this case one expects a better energy resolution than for the HELLAZ case and, therefore, an enhanced sensitivity on the parameter $\gamma$ is expected. A detailed analysis of this case is now in progress [18].

In summary, we conclude that a new generation of low-energy solar neutrino-type detectors using strong radioactive neutrino sources may open new experimental possibilities in testing the structure of the electroweak interaction.

We would like to thank useful discussions with Rita Bernabei, Miguel Angel García-Jareño, Con- cha González-García, Philippe Gorodetzky, Francis von Feilitzsch, Sandra Malvezzi, Stanislav Mikheev, Stephan Schonert, and Tom Ypsilantis. This work was supported by DGICYT under grant number PB95-1077 by the TMR network grant ERBFMRXCT960090 of the European Union. O. G. M. was supported by a CONACYT fellowship from the mexican government, and V. S. by the sabbatical grant SAB95506 and RFFR gronts 97-02-16501, 95-02-03724.

\section{REFERENCES}

1. Allen et. al., Phys. Rev. Lett. 55 (1985) 2401.

2. Allen et. al., Phys. Rev. D47 (1993) 11.

3. Vogel and Engel, Phys. Rev. D39 (1989) 3378.

4. GALLEX Coll., Phys. Lett. B342 (1995) 440.

5. R. Bernabei, these proceedings; R. Bernabei et. al., Phys. Lett. B389 (1996) 757; I. R. Barabanov et. al., ROM2F-97/21, submitted to Astroparticle Physics.

6. J. B. Benziger et. al., A proposal for participating in the Borexino solar neutrino experiment, October 30, 1996.

7. F. Arzarello et. al.: CERN-LAA/94-19.

8. A. Alessandrello et al Astropart. Phys. 3 (1995) 239

9. D. Stickland, Electroweak results from $e^{+} e^{-}$ colliders, these proceedings.

10. John N. Bahcall, Marc Kamionkowski, Alberto Sirlin, Phys. Rev. D51 (1995) 6146.

11. J.C. Pati and A. Salam, Phys. Rev. D10 (1975) 275.

R.N. Mohapatra and J.C. Pati, Phys. Rev. D11 (1975) 2558.

12. R.N. Mohapatra and G. Senjanović, Phys. Rev. D23 (1981) 165 and references therein.

13. Barry R. Holstein, Weak Interactions in Nuclei, Princeton series in physics, Princeton University Press, 1989.

14. L. Wolfenstein, Phys. Rev. D29 (1984) 2130.

15. O. Adriani et. al., L3 Coll., Phys. Lett. B306 (1993) 187.

16. M. Cvetic, P. Langacker, hep-ph/9707451.

17. P. Vilain, et. al., CHARM II Coll., Phys. Lett. B332 (1994) 465.

18. O. G. Miranda, V. Semikoz, J. W. F. Valle, in preparation. 